\title{
Optical rotation of heavy hole spins by non-Abelian geometrical means
}

\author{
Hui Sun ${ }^{1,2}$, Xun-Li Feng ${ }^{1,2}$, Chunfeng $\mathrm{Wu}^{1,2}$, Jinming Liu ${ }^{4,1}$, Shangqing Gong ${ }^{3}$, and C. H. $\mathrm{Oh}^{1,2}$ \\ 1 Centre for Quantum Technologies, National University of Singapore, 3 Science Drive 2, Singapore 117543 \\ 2 Department of Physics, National University of Singapore, 2 Science Drive 3, Singapore 117542 \\ 3 State Key Laboratory of High Field Laser Physics, \\ Shanghai Institute of Optics and Fine Mechanics, \\ Chinese Academy of Sciences, Shanghai 201800, China \\ 4 Physics Department, East China Normal University, Shanghai, China
}

(Dated: November 8, 2018)

\begin{abstract}
A non-Abelian geometric method is proposed for rotating of heavy hole spins in a singly positive charged quantum dot in Voigt geometry. The key ingredient is the delay-dependent non-Abelian geometric phase, which is produced by the nonadiabatic transition between the two degenerate dark states. We demonstrate, by controlling the pump, the Stokes and the driving fields, that the rotations about $y$ - and $z$-axes with arbitrary angles can be realized with high fidelity. Fast initialization and heavy hole spin state readout are also possible.

PACS numbers: 78.67.Hc, 03.67.Lx, 03.65.Vf
\end{abstract}

\section{INTRODUCTION}

Electron spins in quantum dots (QDs) are promising candidate for implementations of qubits [1, 2, 3] because of their potential integration into microtechnology. The two spin states of electron can be mapped directly to the two operational states in quantum information processing (QIP). A key element for spinbased QIP is the coherent manipulation of the spin states [4, 5, 6, 7, 8, 9, 10, 11, 12, 13, 14, 15, 16, 17, 18]. This QIP approach requires not only rotation of unknown spin states-the heart of spin-based QIP, but also the spin states initialized in a known state and readout of spins. There has been significant experimental progress in the demonstration of the key DiVincenzo requirements [19], for examples, efficient optical methods for initialization and readout of spins [20, 21, 22, 23, 24]. Significant theoretical and experimental effort has been invested in optical manipulation of electron spin such as by using two Raman-detuned laser pulses 10], Abelian geometric phase induced by $2 \pi$ pulses [12], resonant radiofrequency pulses [13], and so on. Using ultrafast optical pulses, Press et al. reported that they have controlled and observed the spin of a single electron in a semiconductor (over six Rabi oscillations between the two spin states) [4]. Subsequently, the rotations of electron spins about arbitrary axes in a few picoseconds were also demonstrated in an ensemble of QDs [11].

In spin-based QIP, in addition to preparing the spin in a precisely defined state, this state should survive long enough to allow its manipulations. Therefore a long spin coherence time is necessary. Different from the conduction electron, a valence hole has an atomic $p$ orbital, which has negligible overlap with the nuclei. Consequently, the suppressed hyperfine interaction leads to a longer spin coherence time than that of electron. This may provide an attractive route to hole-spin-based applications free from the complications caused by the fluctuating nuclear spin system. In particular, Heiss et al. reported that the spin-relaxation times of holes are up to 270 microseconds in InGaAs QDs embedded in a GaAs diode structure 25]. Besides long coherence times, an equally important requirement is the ability to manipulate spins coherently. More recently, the high-fidelity hole spin initialization by optical pumping [26], optical control and readout of hole spin 27] have been demonstrated experimentally. These works promote the spin of a hole in a semiconductor QD to be the best position to be a contender for the role of a solid-state qubit.

When a quantum system governed by a Hamiltonian with nondegenerate eigenstates undergoes some appropriate cyclic evolutions by adiabatically changing the controllable parameters, besides a dynamical phase, it may acquire a so-called geometric phase or Berry phase [28]. Wilczek and Zee generalized the geometric phase to degenerate systems, i.e., non-Abelian geometric phase [29]. The geometric phase differs from the dynamic phase in that the former depends only on the geometry of the path executed, being therefore insensitive to the local inaccuracies and fluctuations. They are thus expected to be particularly robust against noise [30, 31, 32, 33, 34, 35].

Motivated by these work, we propose a method for manipulating arbitrary rotation of an unknown heavy hole $(\mathrm{HH})$ spin state in a singly positive charged quantum dot. By applying an external magnetic field in Voigt geometry, a double tripod-shaped scheme can be configured. Most importantly, in contrast to the existing proposals based on electron spin, the $\mathrm{HH}$ spin rotations are realized in terms of the non-Abelian Berry phase, which is acquired by controlling the parameters along adiabatic loops, i.e., stimulated Raman adiabatic passage (STIRAP) 36] and fractional STIRAP [37]. The STIRAP process can be used to transfer populations coherently between quantum states through "dark state" which efficiently suppress relaxation. The geometric phases accumulated during a STIRAP process were previously investigated for tripod systems [38] and double- $\Lambda$ systems [39]. 
In this paper, after briefly reviewing non-Abelian geometric phase (Sec. III), we discuss the hole and electron energy levels of a singly positive charged QD in Voigt geometry and the selection rules in Sec. IIII and study the feasibility of initialization by optical pumping. In Sec. IV we show how to achieve a two-fold degenerate dark states, and how to implement the rotations about $y$ - and $z$-axes by using the non-Abelian geometric phase produced by the nonadiabatically coupling between the two degenerate dark states. The fidelities of these rotations are also discussed in this section. The readout of spin state is discussed in Sec. V] In Sec. [VI we end with some remarks.

\section{NON-ABELIAN GEOMETRIC PHASE}

Our propositions of rotating the HH spin about $y$ - and $z$-axes are based on non-Abelian geometric phase [29], so we start by recalling the basic facts about non-Abelian geometric phase [30]. We consider an $n$-fold degenerate eigenspace of a Hamiltonian $H\left(\chi_{\kappa}\right)(\kappa=1,2, \cdots, N)$ (i.e., the eigenspace information encoded) depending continuously on parameters $\chi_{\kappa}$. Based on the timedependent Schrödinger equation, we control the parameters along loops $\mathcal{O}$ in an adiabatic fashion so that the initial preparation can evolve according to

$$
|\Psi(t)\rangle=U(\mathcal{O})_{A}|\Psi(t=0)\rangle .
$$

The transformation can be computed in terms of the Wilczek-Zee gauge connection [29]:

$$
U(\mathcal{O})_{A}=\mathcal{P} \exp \oint_{\mathcal{O}} \sum_{\kappa=1}^{N} A_{\kappa} d \chi_{\kappa} .
$$

$\mathcal{P}$ denotes the path-order operator. $A_{\kappa}^{a b}$ is called the gauge potential given by

$$
A_{\kappa}^{a b}=\left\langle\psi^{a}(\chi)\left|\frac{\partial}{\partial \chi_{\kappa}}\right| \psi^{b}(\chi)\right\rangle
$$

with $\left\{\left|\psi^{a}(\chi)\right\rangle\right\}_{a=1}^{n}$ being an orthonormal basis of the degenerate eigenspace. It is worth noting that the parameter-dependent Hamiltonian should evolve adiabatically so that the instantaneous state $|\Psi(t)\rangle$ does not overflow the state vector space spanned by $\left|\psi^{a}\right\rangle$. An intriguing feature of the gauge potential $A$ lies in that it depends only on the geometry of executed path in the space of degenerate states.

\section{THE ENERGY LEVELS AND INITIALIZATION}

In the scheme of rotating $\mathrm{HH}$ spin, the basic idea is to reconfigure a multi-level system with interacting Hamiltonian possessing two-fold degenerate dark states. By

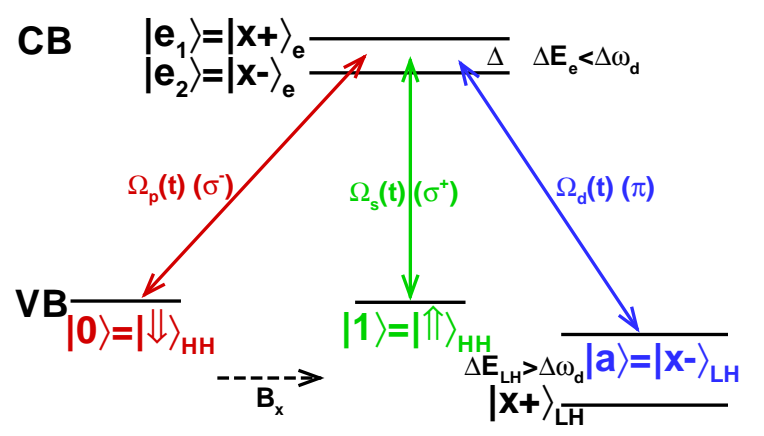

FIG. 1: (Color online) An energy-level diagram of a singly positive charged quantum dot in Voigt geometry together with optical spin selection rules: a degenerate heavy hole $(\mathrm{HH})$ spin states and two eigenstates of nondegenerate electron and light hole (LH) spin states. Solid arrows, respectively, indicate dipole allowed optical transitions driven by the pump $\left(\Omega_{p}(t)\right)$, the Stokes $\left(\Omega_{s}(t)\right)$, the driving $\left(\Omega_{d}(t)\right)$ fields with $\sigma^{+}, \sigma^{-}$and $\pi$ denoting right-hand, left-hand circular, and linear-polarized fields. The photon bandwidth $\left(\Delta \omega_{d}\right)$ should be larger than the electron Zeeman splitting $\Delta E_{\mathrm{e}}$ but smaller than the LH Zeeman splitting $\left(\Delta E_{\mathrm{LH}}\right)$.

changing the Rabi frequencies in adiabatic fashion, we perform a loop in the parameter space. At both the beginning and the end of the cycle, we have only the $\mathrm{HH}$ spin states (up and down). But after a loop a non-Abelian geometric phase is accumulated at $\mathrm{HH}$ spin states. Based upon this property, arbitrary rotations of $\mathrm{HH}$ spin can be implemented.

We consider a singly positive charged GaAs/AlGaAs QD with growth direction $z$, which can be formed naturally by interface steps in narrow quantum well $(\mathrm{QW})$. The electron and holes become localized into QD regions of the QW. In the absence of magnetic field, the lowest conduction-band (CB) level is two-fold degenerate with respect to the spin projection $\pm 1 / 2$. In the valence-band (VB), the hole has a total angular momentum of $3 / 2$, with the projection $m_{J}= \pm 1 / 2$ ("light hole" LH) doublet separated by more than $30-50 \mathrm{meV}$ from the $m_{J}= \pm 3 / 2(\mathrm{HH})$ states due to confinement. It is very large compared to the bandwidth of the picosecond and femtosecond pulsed laser, so one should be able to separate the $\mathrm{HH}$ and $\mathrm{LH}$ excitations by picosecond pulsed fields in practical applications. The spin states of $\mathrm{HH}$ (spin up and down) trapped in the QD, which are denoted by $|0\rangle=|\Downarrow\rangle=\left|\frac{3}{2}, \frac{3}{2}\right\rangle$ and $|1\rangle=|\Uparrow\rangle=\left|\frac{3}{2},-\frac{3}{2}\right\rangle$, are our qubit degrees of freedom. We will perform sequentially the optical initialization, rotations of this spin by non-Abelian geometrical means, and readout of a single hole spin. With $\sigma^{-}$and $\sigma^{+}$excitations, the only dipole allowed optical transitions from the valence $\mathrm{HH}$ states to the conduction electron states are $\left|\frac{3}{2}, \frac{3}{2}\right\rangle \rightarrow\left|\frac{1}{2}, \frac{1}{2}\right\rangle$ and $\left|\frac{3}{2},-\frac{3}{2}\right\rangle \rightarrow\left|\frac{1}{2},-\frac{1}{2}\right\rangle$, and the light hole states cannot be excited because of the frequency selection. The angular moment restriction inhibits optical coupling between the two HH spin states. Consequently, the structure can be 

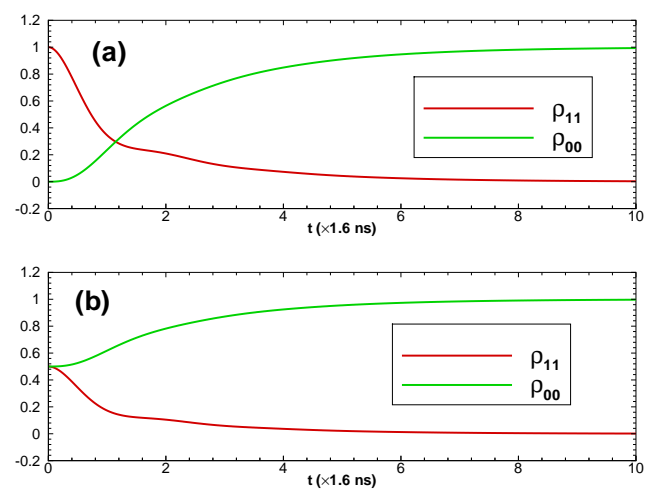

FIG. 2: (Color online) The population of the states $|0\rangle$ and $|1\rangle$ as a function of time with $\sigma^{-}$continuous illumination. Different initial population distributions are considered: (a) $\rho_{00}(t=0)=0, \rho_{11}(t=0)=1.0$; (b) $\rho_{00}(t=0)=\rho_{11}(t=$ $0)=0.5$. The values of parameters are explained in the text.

described by two degenerate independent two-level systems, and the spin flip Raman scattering transitions are ideally dark [23]. The dark transitions should become bright so as to implement spin state initialization, rotations, and readout. The problem can be solved by applying a magnetic field in the Voigt geometry $(x$ direction). The magnetic field lifts the Kramer degeneracy of the LH and electron and reconfigures the eigenstates as $|x \pm\rangle_{\mathrm{LH}}=\left(\left|\frac{3}{2}, \frac{1}{2}\right\rangle \pm\left|\frac{3}{2},-\frac{1}{2}\right\rangle\right) / \sqrt{2}$, and $|x \pm\rangle_{\mathrm{e}}=$ $\left(\left|\frac{1}{2}, \frac{1}{2}\right\rangle \pm\left|\frac{1}{2},-\frac{1}{2}\right\rangle\right) / \sqrt{2}$ (parallel or antiparallel to the magnetic field direction $x$ ) while keeping the degeneracy of the HH with a negligibly small in-plane $g$ factor [7, 40]. The HH spin states remain unaffected by the magnetic field. A double tripod-shaped system is therefore configured, and the energy level diagram and the associated selection rules are represented in Fig. 11. In the rotations of the spin state, the state $|x-\rangle_{\mathrm{LH}}$ is used as an ancillary level, and it is presented by $|a\rangle$. The transitions from the VB states $|0\rangle,|1\rangle$, and $|a\rangle$ to the $\mathrm{CB}$ states $\left|e_{1,2}\right\rangle$ can be excited by the pump ( $\sigma^{-}$-polarized), the Stokes $\left(\sigma^{+}\right.$-polarized $)$, and the driving ( $\pi$-polarized) fields, respectively [41]. The dark transitions therefore become bright and it is possible to initialize and rotate the $\mathrm{HH}$ spin states. The photon bandwidth of the driving field $\left(\Delta \omega_{d}\right)$ can be controlled in a way such that it is larger than the electron Zeeman splitting $\Delta E_{\mathrm{e}}$ but smaller than the LH Zeeman splitting $\left(\Delta E_{\mathrm{LH}}\right)$ [40], the excitation of state $|x+\rangle_{\text {LH }}$ can be thus ignored safely.

The initialization of $\mathrm{HH}$ spin can be accomplished by optical pumping [17, 26]. For example, the spin up $\mathrm{HH}$ state can be prepared by only applying the Stokes field. The electron is excited from the VB band state to two CB band states $\left|e_{1}\right\rangle$ or $\left|e_{2}\right\rangle$, and one spin up HH is left. Exciton (electron-hole pair) recombination occurs between the $\mathrm{CB}$ band electron and the $\mathrm{VB} \mathrm{HH}$ with spin down or up, while the electron recombined with $|\Uparrow\rangle$ hole will be excited again. Since the pump field has not been applied, the resident hole remains in spin up state $|\Uparrow\rangle$.
Conversely, the $\mathrm{HH}$ can also be prepared in spin down state $|\Downarrow\rangle$ by only applying the pump field. To assess the efficiency of optical spin preparation, we have performed numerical simulations using the Liouville equation for the density matrix for the four-level system as shown in Fig. 2, We have assumed that four exciton recombining channels (from two CB electron states to two spin states) proceed incoherently, and they are equal to each other $(1 / 2 \gamma=800$ ps [26]). The magnetic field and the spin-flip rates for hole and electron are taken as $B_{x}=55 \mathrm{mT}, \gamma_{h h}^{-1}=\gamma_{e e}^{-1}=1 \mathrm{~ms}$ [26], and the Rabi frequencies as $1.0 \gamma$. The $\mathrm{HH}$ spin state initialization with a fidelity close to $1[\sim 99.95 \%$, we define the fidelity of the hole spin initialization as $\left(\rho_{00}-\rho_{11}\right) /\left(\rho_{00}+\rho_{11}\right)$ for $\sigma^{+}$ polarization with $\rho_{00}\left(\rho_{11}\right)$ being the population of state $|0\rangle(|1\rangle)]$ is possible to be achieved within a few times the inverse of the exciton recombining rate $(\sim 1.6 \mathrm{~ns})$ in the QD structure in Voigt geometry. The initialization of $\mathrm{HH}$ spin here is realized based on the fact that the magnetic field applied in Voigt geometry reconfigures the electron eigenstates, which is different from Refs. [17, 26], where the initializations are realized based on spin precession.

\section{ARBITRARY ROTATIONS BY NON-ABELIAN GEOMETRICAL MEANS}

It is well known that, with two noncommutable rotations about two axes, any rotation can be implemented as a composite rotation [42]. Here we design two noncommutable rotations about $y$ - and $z$-axes and compose general rotations from them. In the following, based on nono-Alelian geometric phase, we first show how to rotate the $\mathrm{HH}$ spin about $y$-axis, and then explain how to control the rotation about $z$-axis with the relative phase between the Stokes and the driving fields. As a result, any rotations of the $\mathrm{HH}$ spin can be realized.

\section{A. Rotation about $y$-axis}

In order to rotate the $\mathrm{HH}$ spin about $y$-axis, we apply the pump field, the Stokes field and the driving field to excite the corresponding transitions (as shown in Fig. 11). The Hamiltonian in the interaction picture and in the rotating-wave approximation (RWA) is given by

$$
\begin{aligned}
H(t)= & \hbar\left[\left(\Delta_{s}-\Delta_{p}\right)|1\rangle\left\langle 1\left|+\left(\Delta_{d}-\Delta_{p}\right)\right| a\right\rangle\langle a|\right. \\
& -\Delta_{p}\left|e_{1}\right\rangle\left\langle e_{1}\left|-\left(\Delta_{p}+\Delta\right)\right| e_{2}\right\rangle\left\langle e_{2}\left|-\Omega_{p 1}(t)\right| e_{1}\right\rangle\langle 0| \\
& -\Omega_{s 1}(t)\left|e_{1}\right\rangle\left\langle 1\left|-\Omega_{d 1}(t)\right| e_{1}\right\rangle\left\langle a\left|-\Omega_{p 2}(t)\right| e_{2}\right\rangle\langle 0| \\
& \left.-\Omega_{s 2}(t)\left|e_{2}\right\rangle\left\langle 1\left|-\Omega_{d 2}(t)\right| e_{2}\right\rangle\langle a|\right]+ \text { H.c. },
\end{aligned}
$$

where the half Rabi frequency is defined as $\Omega_{j k}(t)=$ $\left\langle e_{k}\left|\vec{\mu} \cdot \vec{E}_{j}(t)\right| q\right\rangle / 2 \hbar$ with $\vec{\mu}$ being the dipole moment, $k=1,2, j=p(s, d)$ denoting the pump (the Stokes and the driving) field, and $q=0,1, a . \Delta_{j}=\omega_{j}-\left(\omega_{e 1}-\omega_{q}\right)$ is the detuning and $\Delta=\omega_{e_{1}}-\omega_{e_{2}}=\left|g_{x}^{e}\right| \mu_{B} B_{x} / \hbar$ is the elec- 
tron Zeeman splitting with $g_{x}^{e}$ and $\mu_{B}$ representing Landé factor of electron and Bohr magneton, respectively.

When the pump, the Stokes, and the driving fields are tuned to match the conditions $\Delta_{p}=\Delta_{s}=\Delta_{d} \neq-\Delta / 2$ (three fields are tuned to three-photon resonance, but they are not at the middle point of the two electron Zeeman splitting levels) and $\Omega_{j 1}(t) / \Omega_{j 2}(t)=C$ (for simplicity, we choose $C=1$ and denote $\Omega_{j 1}(t)=\Omega_{j 2}(t)=$ $\Omega_{j}(t)$, one can easily find from the interaction Hamiltonian (4) that the interacting system has two degenerate dark states

$$
\begin{aligned}
\left|D_{1}\right\rangle= & \cos \theta(t)|1\rangle-\sin \theta(t)|a\rangle, \\
\left|D_{2}\right\rangle= & \cos \varphi(t)|0\rangle-\sin \varphi(t) \sin \theta(t)|1\rangle \\
& -\sin \varphi(t) \cos \theta(t)|a\rangle,
\end{aligned}
$$

where the mixing angle $\theta(t)$ and the additional mixing angle $\varphi(t)$ are defined as

$$
\tan \theta(t)=\frac{\Omega_{s}(t)}{\Omega_{d}(t)}, \quad \tan \varphi(t)=\frac{\Omega_{p}(t)}{\sqrt{\Omega_{s}^{2}(t)+\Omega_{d}^{2}(t)}} .
$$

It is well know that the exciton recombination occurs only if there is electron excited to the $\mathrm{CB}$ states $\left|e_{1,2}\right\rangle$. The two-fold degenerate dark states $\left|D_{1,2}\right\rangle$, which are known as trapped states, receive no contributions from the CB electron states [see Eqs. (5) and (6)]. Hence the rotation of $\mathrm{HH}$ spin about $y$-axis is robust against the exciton recombination process, and thus leading to high fidelity operations. It is also worth noting that, in the absence of the three fields, i.e., all the parameters (actually the angles $\theta(t)$ and $\varphi(t))$ are fixed to zero, the previous eigenstates coincide with the two spin states $\left|D_{1}(0)\right\rangle=|\Uparrow\rangle$ and $\left|D_{2}(0)\right\rangle=|\Downarrow\rangle$. When the three fields are applied adiabatically and hence the angles $\theta(t)$ and $\varphi(t)$ change adiabatically, the non-Abelian geometric connection components can be calculated, according to Eq. (3), and we have

$$
A=A_{\theta} d \theta=-i \sin \varphi(t) \sigma_{y} d \theta,
$$

with $\sigma_{y}$ being the $y$-component Pauli matrix. The related unitary operation is

$$
U(\mathcal{O})=\exp \left(-i \sigma_{y} \int_{\mathcal{O}} \sin \varphi(t) d \theta\right)=R_{y}(\beta)
$$

where the rotating angle $\beta$ is given by

$$
\beta=\int_{\mathcal{O}} \sin \varphi(t) d \theta
$$

Similar degenerate dark states and non-Alelian geometric connection have been realized for ion trap [32] and atoms [30, 38]. However, in the QD structure under consideration, the non-Abelian geometric connection $A$ is based on $\mathrm{HH}$ spin states.

For a quantitative analysis of the rotating angle $\beta$ about $y$-axis, we assume that the pump, the Stokes

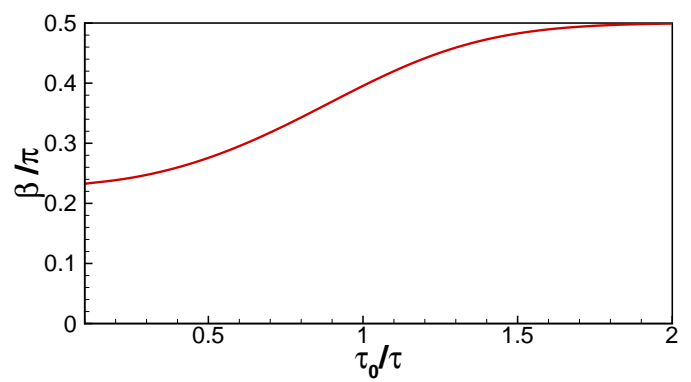

FIG. 3: (Color online) The rotating angle $\beta$ about $y$-axis in units of $\pi$ as a function of the delay $\tau_{0}$. The values of parameters are explained in the text.

and the driving fields have Gaussian shapes as $\Omega_{d}(t)=$ $\Omega_{d}^{0} \exp \left[-\left(t+\tau_{0}\right)^{2} / \tau^{2}\right], \quad \Omega_{p}(t)=\Omega_{p}^{0} \exp \left(-t^{2} / \tau^{2}\right)$, and $\Omega_{s}(t)=\Omega_{s}^{0} \exp \left[-\left(t-\tau_{0}\right)^{2} / \tau^{2}\right]$ with $\tau$ and $\tau_{0}$ being, respectively, the pulse widths and the delay. Figure 3 shows that the evolution of the rotating angle $\beta$ about $y$-axis in unit of $\pi$ as a function of delay $\tau_{0}$. In the interaction, the time-dependent Hamiltonian should be performed sufficiently slowly. According to the condition for adiabatic passage [36], we take $\Omega_{p}^{0} \tau=\Omega_{s}^{0} \tau=\Omega_{d}^{0} \tau=50 \gg 1$, which ensures no transition between the dark and bright states (not given in this paper). Figure 3 shows clearly that the rotating angle $\beta$ is delay-dependent. It goes up successively with increasing value of the delay, and then reaches its maximum value, $\pi / 2$, when the delay $\tau_{0}$ is large.

Thus we have shown that the rotation of hole spin about $y$-axis can be implemented by using the nonAbelian geometric phase. The rotation is determined only by the global property and does not depend upon the details of the evolution path in the parameters space.

\section{B. Rotation about $z$-axis}

As suggested in Ref. 32], by setting $\Omega_{p}(t)=0$ and changing adiabatically $\Omega_{s}(t)$ and $\Omega_{d}(t)$, the rotation about $z$-axis can be achieved by making use of the Abelian geometric phase in our QD system. However, by involving the non-Abelian geometric phase, here we suggest another method for rotating the hole spin about $z$-axis. An important feature of this rotation is that the rotating angle about $z$-axis is not geometric phasedependent, it is controlled by the relative phase between the Stokes and the driving fields. To do so, we set $\Omega_{p}(t)=0$ so that the spin down state $|0\rangle$ is decoupled, and assume the phase of the driving field is zero, the relative phase $\phi$ is therefore the phase of the Stokes field. The time-dependent Hamiltonian $H(t)$ in the interaction picture and RWA takes the form

$$
\begin{aligned}
H(t)= & \hbar\left[\left(\Delta_{d}-\Delta_{s}\right)|a\rangle\left\langle a\left|-\Delta_{s}\right| e_{1}\right\rangle\left\langle e_{1}\right|\right. \\
& -\left(\Delta_{s}+\Delta\right)\left|e_{2}\right\rangle\left\langle e_{2}\right|-\Omega_{d}(t)\left(\left|e_{1}\right\rangle\left\langle a|+| e_{2}\right\rangle\langle a|\right) \\
& \left.-\Omega_{s}(t) \exp (-i \phi)\left(\left|e_{1}\right\rangle\left\langle 1|+| e_{2}\right\rangle\langle 1|\right)\right]+ \text { H.c., }(11)
\end{aligned}
$$


where $\phi$ is the relative phase between the Stokes and the drive fields. In the derivation of Hamiltonian (11), the condition $\Omega_{j 1} / \Omega_{j 2}=C=1$ is applied. When the Stokes and the driving fields are controlled to satisfy two photon resonance, only one dark state $\left(\left|D_{1}(t)\right\rangle\right)$ exists. The hole spin cannot be rotated by non-Abelian geometrical means. Fortunately, however, when the two fields are tuned to the middle point of the two electron Zeeman splitting levels, there is another dark state [39]. The two-fold degenerate dark states are

$$
\begin{aligned}
\left|D_{1}(t)\right\rangle= & \cos \theta(t) e^{i \phi}|1\rangle-\sin \theta(t)|a\rangle, \\
\left|D_{2}(t)\right\rangle= & \frac{1}{\sqrt{2}} \cos \varphi(t)\left(\left|e_{1}\right\rangle-\left|e_{2}\right\rangle\right)+\sin \varphi(t) \cos \theta(t)|a\rangle \\
& \quad+\sin \varphi(t) \sin \theta(t) e^{i \phi}|1\rangle,
\end{aligned}
$$

where the mixing angle $\theta(t)$ and the additional mixing angle $\varphi(t)$ related to the electron Zeeman energy splitting $\Delta$ are respectively defined as

$$
\tan \theta(t)=\frac{\Omega_{s}(t)}{\Omega_{d}(t)}, \quad \tan \varphi(t)=\frac{\Delta / 2}{\sqrt{2\left(\Omega_{s}^{2}(t)+\Omega_{d}^{2}(t)\right)}} .(14)
$$

A similar form of double degenerate dark states in double- $\Lambda$ atomic scheme has been obtained [39]. However, in our QD structure the degenerate $\mathrm{HH}$ spin-based dark states stem from electron Zeeman splitting.

In adiabatic limit (the time derivative of the mixing angles $\theta(t)$ and $\varphi(t)$ are small compared with the splitting of eigenvalues, given by $\left.2 \sqrt{2\left(\Omega_{p}^{2}(t)+\Omega_{s}^{2}(t)\right)+(\Delta / 2)^{2}}\right)$, only the transitions between the degenerate dark states $\left|D_{1}(t)\right\rangle$ and $\left|D_{2}(t)\right\rangle$ should be taken into account, and the nonadiabatic coupling of states $\left|D_{1}(t)\right\rangle$ and $\left|D_{2}(t)\right\rangle$ to other states (the expressions have not given in this paper) can be safely ignored [38]. $\left\langle D_{2} \mid \dot{D}_{1}\right\rangle=-\sin \varphi(t) \dot{\theta}(t)$ also exhibits that a nonadidabatic transition between the two-fold degenerate dark states may occur. Although the dark state $\left|D_{2}(t)\right\rangle$ receives contribution from the $\mathrm{CB}$ electron states [see Eq. [13)], we note that the electron magnetic-dependent Zeeman energy splitting is independent of time. At the beginning and end of the adiabatic process, we have $\Delta^{2} \gg \Omega_{s}^{2}(t)+\Omega_{d}^{2}(t)$, which therefore leads to $|\varphi|=\pi / 2$. The CB electron states have no influence on the final dark state $\left|D_{2}(+\infty)\right\rangle$. In other words, there is no electron in the $\mathrm{CB}$ states when the interaction is finished.

Next, we will show how to translate the phase $\phi$ into the $\mathrm{HH}$ spin state $|\Uparrow\rangle$, thus leading to the rotation about $z$-axis with angle $\phi$. Recalling the fact that, in the subspace, $\mathrm{HH}$ is prepared with spin up, namely $\left|D_{1}(0)\right\rangle=|1\rangle$. The Stokes and the driving fields are applied in the counterintuitive order, while they terminate with a constant ratio of their amplitude so that the phase $\phi$ can be introduced into the HH spin state. This extension of STIRAP is called fractional STIRAP, which has been suggested to create the coherent atomic superpositions in a robust way [37]. As shown in Fig. 4(a), the driving field consists of two parts-one with the same
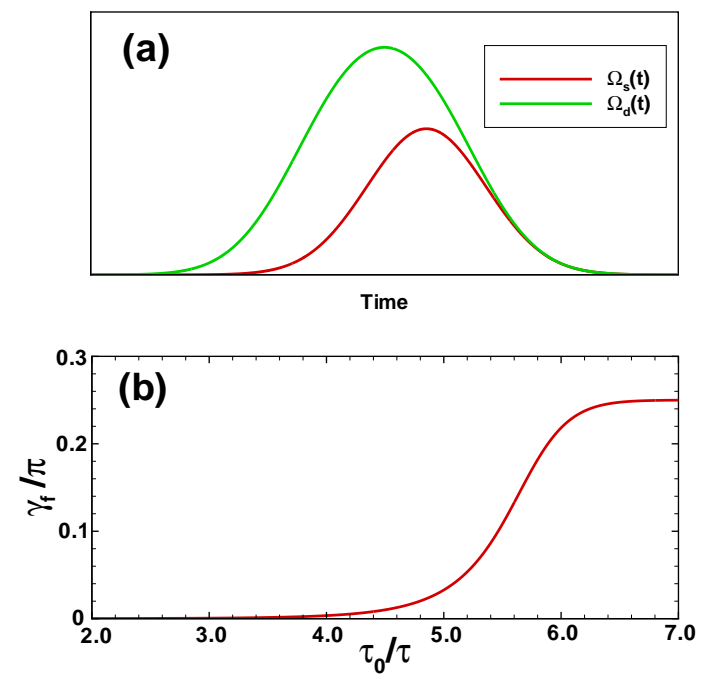

FIG. 4: (Color online) (a) The shapes of the Stokes and the driving fields defined by Eqs. (15) and (16). (b) The geometric phase $\gamma_{f}$ in units of $\pi$ as function of the delay between two pulses $\tau_{0}$ with $\Omega_{p}^{0} \tau=\Omega_{s}^{0} \tau=50$. The values of parameters are explained in the text.

time dependence as the Stokes field and the other coming earlier, for example

$$
\begin{aligned}
& \Omega_{s}(t)=\Omega_{s}^{0} \exp \left(-t^{2} / \tau^{2}\right) \\
& \Omega_{d}(t)=\Omega_{d}^{0}\left\{\exp \left[-\left(t+\tau_{0}\right)^{2} / \tau^{2}\right]+\exp \left(-t^{2} / \tau^{2}\right)\right\}
\end{aligned}
$$

Here $\Omega_{s}^{0}$ and $\Omega_{d}^{0}$ are amplitudes of the Stokes and the driving fields, $\tau$ and $\tau_{0}$ are, respectively, pulse widths and delay between the two parts of driving field. During the interaction, the mixing angle $\theta(t)$ varies from 0 to $\pi / 4$. According to the theory of non-Abelian geometric phase, after the interaction, we have [38, 39]

$$
\begin{aligned}
|\Psi(+\infty)\rangle= & \frac{1}{\sqrt{2}}\left[e^{i \phi}\left(\sin \gamma_{f}+\cos \gamma_{f}\right)|1\rangle\right. \\
& \left.+\left(\sin \gamma_{f}-\cos \gamma_{f}\right)|a\rangle\right],
\end{aligned}
$$

where $\gamma_{f}$ is given by

$\gamma_{f}=\oint_{\mathcal{O}} \frac{\Delta / 2}{\Omega_{p}^{2}(t)+\Omega_{s}^{2}(t)} \frac{\Omega_{s}(t) d \Omega_{p}(t)-\Omega_{p}(t) d \Omega_{s}(t)}{\sqrt{2\left(\Omega_{p}^{2}(t)+\Omega_{s}^{2}(t)\right)+(\Delta / 2)^{2}}}$.

Equation (17) exhibits that the relative phase $\phi$ translates to the HH spin state $|\Uparrow\rangle$ via fractional STIRAP. If $\gamma_{f}$ can be accumulated to $\pi / 4$ by controlling the Stokes and drive fields, after the cycle evolution, the $\mathrm{HH}$ spin will return to the spin up state with the phase $\phi$. As a result, the rotation of $\mathrm{HH}$ spin about $z$-axis is realized, and the varying rotating angle can be obtained by changing the relative phase $\phi$, namely the phase of the Stokes field.

It should be noted that $\gamma_{f}$ is gauge invariant, it depends upon the delay $\tau_{0}[38,39]$. In Fig. [4(b), we plot the 
TABLE I: Fidelity of selected rotations of HH spin.

\begin{tabular}{ccc}
\hline \hline$R_{n}(\phi)$ & $\tau_{0} / \tau$ & Fidelity \\
\hline$R_{y}(\pi / 2)$ & 1.5 & $99.96 \%$ \\
$R_{z}(\pi / 2)$ & 6.5 & $99.99 \%$ \\
$R_{x}(\pi / 2)$ & & $99.94 \%$ \\
\hline \hline
\end{tabular}

evolution of $\gamma_{f}$ in units of $\pi$ as a function of $\tau_{0}$. We take the Rabi frequency amplitudes as: $\Omega_{s}^{0}=\Omega_{d}^{0}=0.5 \mathrm{ps}^{-1}$, the pulse width parameter $\tau=100 \mathrm{ps}$. The magnetic field is taken as $B_{x}=55 \mathrm{mT}$ (we take the in-plane $g$ factor of electron as $g_{x}^{e}=-0.21$ [40], then the electron Zeeman splitting $\Delta \approx 1 \mathrm{GHz}$ ). With these parameters, the adiabatic condition is hold and the governing Hamiltonian evolutes adiabatically. As shown in Fig. प(b) $\gamma_{f}$ increases from 0 by degrees with the growing of the delay, and reaches its maximum value, $\pi / 4$, when the delay $\tau_{0}$ is large. Thus, the relative phase $\phi$ can be used to control the $\mathrm{HH}$ spin rotation about $z$-axis.

By combining the above rotations about $y$ - and $z$ axis, any rotation can be implemented. For example, rotation about $x$-axis can be realized by $R_{x}(\phi)=$ $R_{y}^{\dagger}(\pi / 2) R_{z}(\phi) R_{y}(\pi / 2)$. Our rotation procedure is sensitive to the non-Abelian geometric phase and the relative phase between the Stokes and the driving fields. Moreover, the non-Abelian geometric phase is independent of pulse areas and dependent on the ratios $\tau_{0} / \tau, \Omega_{s}^{0} / \Delta$, and $\Omega_{d}^{0} / \Delta$. Thus it is robust against the fluctuation of the pulse shapes, pulse areas and noise.

\section{Fidelity}

Based on the non-Abelian geometric phase, arbitrary rotations of HH spin are possible. But how about the degree to which our approximate description matches the actual behavior of the system? The fidelity is a measure of how accurate the target gate is implemented and it is defined as $\mathcal{F}(U)=\overline{\left|\left\langle\Psi\left|U^{\dagger} U_{i d}\right| \Psi\right\rangle\right|^{2}}$, where $U_{i d}$ is the target operation, $U$ is the actual operation, and the average is taken over all input spin states [43]. By numerical simulation of the density matrix equations, the fidelity can be calculated. Typical rotation fidelities, listed in Table II are on the order of $99.9 \%$. The rotations of $\mathrm{HH}$ spin are realized with high fidelity.

\section{READOUT}

The accurate measurement of the spin state of each qubit is essential in a quantum computation scheme. In our scheme, the $\sigma^{+}$-polarized Stokes (the $\sigma^{-}$-polarized pump) field can only excite the $\mathrm{HH}$ state with spin down (up). The measurement of the HH spin states therefore can be achieved by applying a $\sigma^{+}$or $\sigma^{-}$polarized continuous laser field. When the $\sigma^{+}$-polarized field is applied, for example, if the spin is rotated to $|\Downarrow\rangle$, the QD will emit a single photon from the $\left|e_{1,2}\right\rangle \rightarrow|\Downarrow\rangle$ transitions, which can be detected using a single-photon counter [4].

\section{CONCLUSIONS}

To summarize, we consider a singly positive charged quantum dot, and demonstrate sequentially the initialization, the optical rotations of $\mathrm{HH}$ spin with nonAbelian geometrical means, and readout of a single hole spin. Together with an magnetic field applied in Voigt geometry, the quantum dot system can be reconfigured as a double tripod scheme. When the pump, the Stokes and the driving fields are tuned to satisfy certain conditions, the QD system has two-fold degenerate dark states. Based on the non-Abelian geometric phase produced by the nonadiabatic coupling between the two dark states, not only can the $\mathrm{HH}$ spin be rotated about $y$-axis with stimulated Raman adiabatic passage, but also the relative phase between the Stokes and the driving fields can be translated into the hole spin state with fractional stimulated Raman adiabatic passages, leading to the implementation of rotation about $z$-axis. Therefore the key step of optical arbitrary rotations of $\mathrm{HH}$ spin with high fidelity for QIP can be implemented by non-Alelian geometrical means. It is in principle useful for spin-based quantum information processing.

\section{Acknowledgments}

This work is supported by the National Research Foundation and Ministry of Education, Singapore under academic research grant No. WBS: R-710-000-008-271. One of the authors (H. S.) would also like to acknowledge the support of the National Basic Research Program of China (973 Program) under Grant No. 2006CB921104, the author (S. Q. G.) acknowledges funding from the National Natural Science Foundation of China through Grant No. 10874194 and the author (J. M. L.) acknowledges funding from the National Natural Science Foundation of China under Grant No. 60708003.
[1] D. Loss and D. P. DiVincenzo, Phys. Rev. A 57, 120 (1998).

[2] A. Imamoğlu, D. D. Awschalom, G. Burkard, D. P. Di-
Vincenzo, D. Loss, M. Sherwin, and A. Small, Phys. Rev. Lett. 83, 4204 (1999).

[3] R. Hanson, L. P. Kouwenhoven, J. R. Petta, S. Tarucha, 
and L. M. K. Vandersypen, Rev. Mod. Phys. 79, 1217 (2007).

[4] D. Press, T. D. Ladd, B. Y. Zhang, and Y. Yamamoto, Nature Phys. 456, 218 (2008).

[5] W. A. Coish and D. Loss, Phys. Rev. B 75, 161302(R) (2007).

[6] R. Hanson and G. Burkard, Phys. Rev. Lett. 98, 050502 (2007).

[7] Yanwen Wu, Erik D. Kim, Xiaodong Xu, Jun Cheng, D. G. Steel, A. S. Bracker, D. Gammon, Sophia E. Economou, and L. J. Sham, Phys. Rev. Lett. 99, 097402 (2007).

[8] S. G. Carter, Z. Chen, and S. T. Cundiff, Phys. Rev. B 76, 201308(R) (2007).

[9] M. V. Gurudev Dutt, Jun Cheng, Yanwen Wu, Xiaodong $\mathrm{Xu}$, D. G. Steel, A. S. Bracker, D. Gammon, S. E. Economou, Ren-Bao Liu, and L. J. Sham Phys. Rev. B 74, 125306 (2006).

[10] C Emary and L J Sham, J. Phys.: Condens. Matter 19, 056203 (2007)

[11] A. Greilich, S. E. Economou, S. Spatzek, D. R. Yakovlev, D. Reuter, A. D. Wieck, T. L. Reinecke, and M. Bayer, Nature Phys. 5, 262 (2009).

[12] S. E. Economou and T. L. Reinecke, Phys. Rev. Lett. 99, 217401 (2007); S. E. Economou, L. J. Sham, Yanwen Wu, and D. G. Steel, Phys. Rev. B 74, 205415 (2006).

[13] K. C. Nowack, F. H. L. Koppens, Yu. V. Nazarov, and L. M. K. Vandersypen, Science 318, 1430 (2007).

[14] J. R. Petta, A. C. Johnson, J. M. Taylor, E. A. Laird, A. Yacoby, M. D. Lukin, C. M. Marcus, M. P. Hanson, A. C. Gossard, Science 309, 2180 (2005).

[15] A. De, C. E. Pryor, and M. E. Flatté, Phys. Rev. Lett. bf 102, 017603 (2009).

[16] T. Andlauer and P. Vogl, Phys. Rev. B 79, 045307 (2009).

[17] C. Galland and A. Imamoğlu, Phys. Rev. Lett. 101, 157404 (2008).

[18] E. Paspalakis, Z. Kis, E. Voutsinas, and A. F. Terzis, Phys. Rev. B 69, 155316 (2004).

[19] D. P. DiVincenzo, Fortschr. Phys. 48, 771 (2000).

[20] M. Atatüre, J. Dreiser, A. Badolato, A. Högele, K. Karrai, and A. Imamoğlu, Science 312, 551 (2006).

[21] C. Emary, Xiaodong Xu, D. G. Steel, S. Saikin, and L. J. Sham, Phys. Rev. Lett. 98, 047401 (2007).

[22] M. Atatüre, J. Dreiser, A. Badolato, and A. Imamoğlu, Nature Phys. 3, 101 (2007).

[23] Xiaodong Xu, Yanwen Wu, Bo Sun, Qiong Huang, Jun Cheng, D. G. Steel, A. S. Bracker, D. Gammon, C. Emary, and L. J. Sham, Phys. Rev. Lett. 99, 097401 (2007).

[24] D. Kim, S. E. Economou, S. C. Bădescu, M. Scheibner, A. S. Bracker, M. Bashkansky, T. L. Reinecke, and D. Gammon, Phys. Rev. Lett. 101, 236804 (2008).
[25] D. Heiss, S. Schaeck, H. Huebl, M. Bichler, G. Abstreiter, J. J. Finley, D. V. Bulaev, and D Loss, Phys. Rev. B 76, 241306(R) (2007).

[26] B. D. Gerardot, D. Brunner, P. A. Dalgarno, P. Öhberg, S. Seidl, M. Kroner, K. Karrai, N. G. Stoltz, P. M. Petroff, and R. J. Warburton, Nature (London) 451, 441 (2008).

[27] A. J. Ramsay, S. J. Boyle, R. S. Kolodka, J. B. B. Oliveira, J. Skiba-Szymanska, H. Y. Liu, M. Hopkinson, A. M. Fox, and M. S. Skolnick, Phys. Rev. Lett. 100, 197401 (2008).

[28] M. V. Berry, Proc. R. Soc. London, Ser. A 392, 45 (1984).

[29] F. Wilczek and A. Zee, Phys. Rev. Lett. 52, 2111 (1984).

[30] P. Zanardi and M. Rasetti, Phys. Lett. A 264, 94 (1999); J. Pachos, P. Zanardi, and M. Rasetti, Phys. Rev. A 61, 010305(R) (1999).

[31] A. Recati, T. Calarco, P. Zanardi, J. I. Cirac, and P. Zoller, Phys. Rev. A 66, 032309 (2002).

[32] L. M. Duan, J. I. Cirac, and P. Zoller, Science 292, 1659 (2001).

[33] S. L. Zhu and Z. D. Wang, Phys. Rev. Lett. 91, 187902 (2003); X. D. Zhang, Z. D. Wang, L. B. Hu, Z. M. Zhang, and S. L. Zhu, New J. Phys. 10, 043031 (2008).

[34] Xun-Li Feng, Zisheng Wang, Chunfeng Wu, L. C. Kwek, C. H. Lai, and C. H. Oh, Phys. Rev. A 75, 052312 (2007); C.-Y. Chen, M. Feng, X.-L. Zhang, and K.-L. Gao, Phys. Rev. A 73, 032344 (2006).

[35] P. Solinas, P. Zanardi, N. Zanghì, and F. Rossi, Phys. Rev. A 67, 062315 (2003).

[36] K. Bergmann, H. Theuer, and B. W. Shore, Rev. Mod. Phys. 70, 1003 (1998).

[37] N V Vitanov, K-A Suominen, and B W Shore, J. Phys. B: At. Mol. Opt. Phys. 32, 4535 (1999).

[38] R. G. Unanyan, B. W. Shore, and K. Bergmann, Phys. Rev. A 59, 2910 (1999); R. G. Unanyan, and M. Fleischhauer, Phys. Rev. A 69, 050302(R) (2004).

[39] Shiqi Jin, Shangqing Gong, Ruxin Li, and Zhizhan Xu, Phys. Rev. A 69, 023408 (2004); Shangqing Gong, R. G. Unanyan, and K. Bergmann, Eur. Phys. J. D 19, 257 (2002).

[40] H. Kosaka, H. Shigyou, Y. Mitsumori, Y. Rikitake, H. Imamura, T. Kutsuwa, K. Arai, and K. Edamatsu, Phys. Rev. Lett. 100, 096602 (2008).

[41] Optical Orientation, edited by F. Meier and B. P. Zakharchenya (Elsevier, Amsterdam, 1984).

[42] A. Barenco, C. H. Bennett, R. Cleve, D. P. DiVincenzo, N. Margolus, P. Shor, T. Sleator, J. A. Smolin, and H. Weinfurter, Phys. Rev. A 52, 3457 (1995).

[43] M. D. Bowdrey, D. K. L. Oi, A. J. Short, K . Banaszek, and J. A. Jones, Phys. Lett. A 294, 258 (2002). 